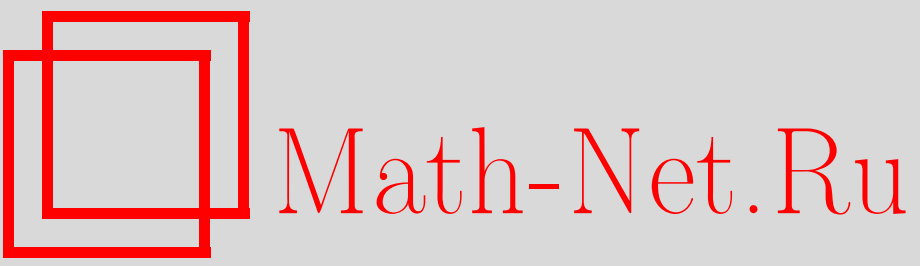

А. С. Рыбаков, Оценки количества чисел со специальным разложением на простые множители, Матем. вопр. криптогр., 2016, том 7, выпуск 1, 119-142

DOI: https://doi.org/10.4213/mvk178

Использование Общероссийского математического портала Math-Net.Ru подразумевает, что вы прочитали и согласны с пользовательским соглашением

http://www.mathnet.ru/rus/agreement

Параметры загрузки:

IP: 34.227 .88 .159

26 апреля 2023 г., 06:29:27 
МАТЕМАТИЧЕСКИЕ ВОПРОСЫ КРИПТОГРАФИИ

2016 T. 7 № 1 C. 119-142

УДК 511.333

\title{
Оценки количества чисел \\ со специальным разложением на простые множители
}

\author{
А. С.Рыбаков
}

Лаборатории «ТВП», Москва

Получено 20.IV.2015

В работе найдены интегральные формулы для некоторых обобщений известной функции Дикмана, позволяющие оценивать количество целых чисел из интервала большой длины, разлагающихся на простые множители со специфическими ограничениями. Эти формулы имеют пониженную кратность интегрирования и обобщают ранее известные формулы из работ Р. Ламберта, В. Х. Эккелькамп, а также автора.

Работа выполнена при финансовой поддержке Академии криптографии Российской Федерации.

Ключевые слова: функция Дикмана, факторизация, оценка числа соотношений просеивания

Estimates of the number of integers with the special prime factorization

\section{A. S. Rybakov}

TVP Laboratories, LLC, Moscow

Abstract. We obtain several integral formulas for some generalizations of the known Dickman function which makes it possible to estimate the number of integers in a long interval with prime factorizations satisfying specific conditions. These formulas contain integrals of reduced multiplicity and generalize the known formulas due to R. Lambert, V.H. Ekkelkamp and the author are generalized.

Keywords: Dickman function, integer factorization, estimates for the number of the sieving equations

Citation: Mathematical Aspects of Cryptography, 2016, v. 7, № 1, pp. 119-142 (Russian)

(c) Академия криптографии Российской Федерации, 2016 г. 


\section{1. Введение}

В работе выводятся удобные интегральные формулы для эффективного вычисления некоторых обобщений хорошо известной функции Дикмана [5]. Точнее, речь будет идти о функциях $G_{m}\left(\beta_{0}, \alpha_{1}, \beta_{1}, \alpha_{2}, \beta_{2}\right), m=1,2, \ldots$, равных предельной при $x \rightarrow \infty$ вероятности того, что все простые делители случайно выбранного из отрезка $[1 ; x]$ целого числа удовлетворяют неравенству $p<x^{\beta_{0}}$, за исключением ровно $m$ больших простых делителей $q$ (с учетом кратности), из которых первый (наименьший) большой простой делитель принадлежит промежутку $\left[x^{\alpha_{1}} ; x^{\beta_{1}}\right)$, а остальные большие простые делители промежутку $\left[x^{\alpha_{2}} ; x^{\beta_{2}}\right)$. Такие числа в теории факторизации обычно называются строго $\left(x^{\beta_{0}}, x^{\alpha_{1}}, x^{\beta_{1}}, x^{\alpha_{2}}, x^{\beta_{2}}, m\right)$-полугладкими.

Поискам способов быстрого вычисления частных случаев функций $G_{m}$ при некоторых значениях $m$ и ограничениях на числа $\alpha_{i}$ и $\beta_{i}$ был посвящен целый ряд работ. В основе всех алгоритмов, предложенных в этих работах, лежат найденные авторами представления функций $G_{m}$ в виде интегралов кратности $m$ (при $m=1$ ) или $m-1$ (при $m \geqslant 2$ ).

В частности, для случая $m=1$ и $\alpha_{1}=\beta_{0}$ быстрый алгоритм нахождения значений $G_{m}$ был предложен в 1996 г. в работе Э. Баха и Р. Перальты [3], являющейся по существу исходной точкой развития всех дальнейших методов, касающихся функций более сложного вида. Этот алгоритм требует $O(K \ln K)$, где $K=\left\lceil\beta_{m} / \beta_{0}\right\rceil+1$, арифметических операций с плавающей точкой и $O(1)$ операций нахождения значения логарифма.

Случай $m=2, \alpha_{1}=\alpha_{2}=\beta_{0}<\beta_{1}=\beta_{2}$ был рассмотрен в диссертации Р. Ламберта [6], опубликованной также в 1996 г.: в [6] показано, что при том же значении $K$ нахождение величины $G_{2}\left(\beta_{0}, \beta_{0}, \beta_{1}, \beta_{0}, \beta_{1}\right)$ требует $O\left(K \ln ^{3} K\right)$ операций сложения и умножения с плавающей точкой, $O(\ln K)$ операций деления и $O(K)$ операций нахождения значений логарифма и дилогарифма

$$
\operatorname{dilog}(x)=\int_{1}^{x} \frac{\ln t}{1-t} d t
$$

Эти оценки были получены на основе найденного Р. Ламбертом представления функции $G_{2}$ в виде интеграла кратности 1 (базовая интегральная формула для $G_{2}$ имеет кратность 2).

Обобщения на случай $m=2, \alpha_{1}=\alpha_{2}=\beta_{0}<\beta_{1}<\beta_{2}$ алгоритма Р. Ламберта, а также найденных им интегральных формул, были приведены в 2010 г. в диссертации В.Х. Эккелькамп [4]; при этом приведенная выше оценка трудоемкости осталась прежней. 
Автором ([1]) предложен более быстрый алгоритм оценки величин $G_{2}$ и $G_{3}$ в случае $\beta_{0} \leqslant \alpha_{1}=\alpha_{2}<\beta_{1}=\beta_{2}$, требующий лишь $O(K \ln K)$ арифметических операций с плавающей точкой и $O(K)$ операций для нахождения значения логарифма. При этом никакие другие операции в методе не используются, в частности, значения дилогарифма вычислять не нужно.

В основе этого алгоритма лежат найденные автором интегральные формулы для $G_{2}$ (кратности 1) и для $G_{3}$ (кратности 2). Формулы для $G_{2}$, по существу, совпадают с формулами, найденными Р. Ламбертом в более частном случае, однако записываются в унифицированном и удобном для программирования виде.

Отметим, что случай $m=3$ является существенно более трудным, чем случаи $m=1$ и $m=2$, поскольку с ростом $m$ растет и кратность интеграла в формуле вычисления величины $G_{m}$. Базовая формула для $G_{3}$ представляет собой тройной интеграл. Понижение кратности интегрирования и построение алгоритма, аналогичного исходному алгоритму Э. Баха и Р. Перальты, является в данном случае намного более сложной задачей.

Также отметим, что для практических приложений случай произвольных значений параметров $\alpha_{i}$ и $\beta_{i}$ представляет существенно больший интерес, чем случай, когда ряд этих параметров имеет совпадающие значения. Для пояснения данного тезиса надо напомнить, что наиболее быстрым на сегодня методом факторизации больших чисел является метод решета числового поля (GNFS), самым трудоемким этапом данного метода является этап просеивания, а самым эффективным методом просеивания - алгоритм решеточного просеивания Дж. Полларда [7].

В решеточном просеивании рассматриваются простые числа трех типов: простые $p$ из факторной базы, специальные простые $q$ и большие простые $r$, при этом к факторной базе относят все $p<B_{0}$, для специальных простых $q$ выбирается некоторый промежуток $\left[A_{1}, B_{1}\right)$, а большие простые $r$ должны удовлетворять ограничению $q \leqslant r<B_{2}$.

Обычно на практике числа $B_{0}, A_{1}, B_{1}$ и $B_{2}$ всегда различны. Например, для последнего рекорда факторизации (768 бит, см. [2]), установленного в 2010 г., были выбраны следующие значения параметров: $B_{0}=2,0 \cdot 10^{8}, A_{1}=$ $4,5 \cdot 10^{8}, B_{1}=1,1 \cdot 10^{10}$ и $B_{2}=1,6 \cdot 10^{10}$.

Таким образом, рассматриваемая в настоящей работе задача оценки количества $\left(B_{0}, A_{1}, B_{1}, A_{2}, B_{2}, m\right)$-полугладких чисел в большом промежутке точнее соответствует современной практике факторизации больших чисел, чем во всех указанных выше работах предшественников. 


\section{2. Базовые интегральные формулы для функций $G_{2}$ и $G_{3}$}

Обозначим через $\Psi_{m}\left(x, B_{0}, A_{1}, B_{1}, A_{2}, B_{2}\right)$ количество не превосходящих число $x$ натуральных чисел $n$, у которых все простые делители $p$ меньше $B_{0}$, за исключением точно $m$ больших простых делителей $q$ (с учетом кратности), из которых первый (наименьший) большой простой делитель принадлежит промежутку $\left[A_{1}, B_{1}\right)$, а остальные большие простые делители промежутку $\left[A_{2}, B_{2}\right)$. Тогда величина $G_{m}\left(\beta_{0}, \alpha_{1}, \beta_{1}, \alpha_{2}, \beta_{2}\right)$ равна пределу отношения $\Psi_{m}\left(x, x^{\beta_{0}}, x^{\alpha_{1}}, x^{\beta_{1}}, x^{\alpha_{2}}, x^{\beta_{2}}\right) / x$ при $x \rightarrow \infty$.

В данном разделе рассматриваются случаи $m=2$ и $m=3$. Для вывода интересующих нас формул удобно обозначить через $n_{(i)}$ число, равное $i$-му по порядку элементу отсортированного по убыванию набора простых делителей натурального числа $n$, если в этом наборе имеется не менее $i$ элементов с учетом кратности, и 1 в остальных случаях.

Тогда по определению

$$
\begin{aligned}
& \Psi_{2}\left(x, x^{\beta_{0}}, x^{\alpha_{1}}, x^{\beta_{1}}, x^{\alpha_{2}}, x^{\beta_{2}}\right)= \\
& =\sum_{x^{\alpha_{1}} \leqslant p_{1}<x^{\beta_{1}}} \sum_{\max \left\{p_{1}, x^{\alpha_{2}}\right\} \leqslant p_{2}<x^{\beta_{2}}} \#\left\{n \leqslant x: n_{(1)}=p_{2}, n_{(2)}=p_{1}, n_{(3)}<x^{\beta_{0}}\right\}
\end{aligned}
$$

и

$$
\begin{aligned}
& \Psi_{3}\left(x, x^{\beta_{0}}, x^{\alpha_{1}}, x^{\beta_{1}}, x^{\alpha_{2}}, x^{\beta_{2}}\right)=
\end{aligned}
$$

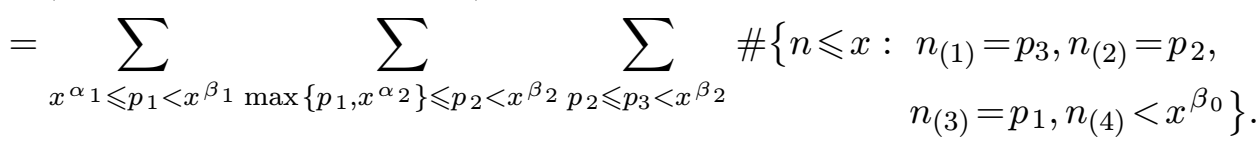

Предположим, что $\beta_{1}+(m-1) \beta_{2}<1$. Заметим, что для практики это условие не является ограничительным, во всех известных рекордах факторизации оно выполнено. Тогда в приведенных выше суммах произведение чисел $p_{1}, p_{2}, \ldots, p_{m}$ всегда меньше, чем $x$. Применяя это к формулам для функций $\Psi_{2}$ и $\Psi_{3}$, получаем, что

$$
\begin{aligned}
& \Psi_{2}\left(x, x^{\beta_{0}}, x^{\alpha_{1}}, x^{\beta_{1}}, x^{\alpha_{2}}, x^{\beta_{2}}\right)= \\
& =\sum_{x^{\alpha_{1}} \leqslant p_{1}<x^{\beta_{1}} \max \left\{p_{1}, x^{\alpha_{2}}\right\} \leqslant p_{2}<x^{\beta_{2}}} \#\left\{k \leqslant \frac{x}{p_{1} p_{2}}: k_{(1)}<x^{\beta_{0}}\right\}= \\
& =\sum_{x^{\alpha_{1}} \leqslant p_{1}<x^{\beta_{1}} \max \left\{p_{1}, x^{\alpha_{2}}\right\} \leqslant p_{2}<x^{\beta_{2}}} \#\left\{k \leqslant \frac{x}{p_{1} p_{2}}: k_{(1)}<\left(\frac{x}{p_{1} p_{2}}\right)^{1 / \widetilde{\beta}\left(p_{1} p_{2}\right)}\right\} .
\end{aligned}
$$


и

$$
\begin{aligned}
& \Psi_{3}\left(x, x^{\beta_{0}}, x^{\alpha_{1}}, x^{\beta_{1}}, x^{\alpha_{2}}, x^{\beta_{2}}\right)= \\
& =\sum_{x^{\alpha_{1}} \leqslant p_{1}<x^{\beta_{1}}} \sum_{\max \left\{p_{1}, x^{\alpha}\right\} \leqslant p_{2}<x^{\beta_{2}}} \sum_{p_{2} \leqslant p_{3}<x^{\beta_{2}}} \#\left\{k \leqslant \frac{x}{p_{1} p_{2} p_{3}}: k_{(1)}<x^{\beta_{0}}\right\}= \\
& =\sum_{x^{\alpha_{1}} \leqslant p_{1}<x^{\beta_{1}}} \sum_{\max \left\{p_{1}, x^{\alpha_{2}}\right\} \leqslant p_{2}<x^{\beta_{2}}} \sum_{p_{2} \leqslant p_{3}<x^{\beta_{2}}} \#\left\{k \leqslant \frac{x}{p_{1} p_{2} p_{3}}:\right. \\
& \left.k_{(1)}<\left(\frac{x}{p_{1} p_{2} p_{3}}\right)^{1 / \widetilde{\beta}\left(p_{1} p_{2} p_{3}\right)}\right\} \text {. }
\end{aligned}
$$

Здесь и ниже для сокращения записи используется обозначение

$$
\widetilde{\beta}(u)=\frac{1}{\beta_{0}}\left(1-\frac{\ln u}{\ln x}\right) .
$$

Пользуясь определением функции Дикмана $\rho(x)$ (см. [3]), можно получить асимптотические равенства

$$
\begin{aligned}
& \Psi_{2}\left(x, x^{\beta_{0}}, x^{\alpha_{1}}, x^{\beta_{1}}, x^{\alpha_{2}}, x^{\beta_{2}}\right) \approx \\
& \quad \approx \sum_{x^{\alpha_{1}} \leqslant p_{1}<x^{\beta_{1}} \max \left\{p_{1}, x^{\alpha_{2}}\right\} \leqslant p_{2}<x^{\beta_{2}}} \frac{x}{p_{1} p_{2}} \rho\left(\widetilde{\beta}\left(p_{1} p_{2}\right)\right)
\end{aligned}
$$

и

$$
\begin{aligned}
& \Psi_{3}\left(x, x^{\beta_{0}}, x^{\alpha_{1}}, x^{\beta_{1}}, x^{\alpha_{2}}, x^{\beta_{2}}\right) \approx \\
& \quad \sum_{x^{\alpha_{1}} \leqslant p_{1}<x^{\beta_{1}}} \sum_{\max \left\{p_{1}, x^{\alpha}\right\}} \sum_{p_{2}<x^{\beta_{2}}} \frac{x}{p_{2} \leqslant p_{3}<x^{\beta_{2}}} \frac{x}{p_{1} p_{2} p_{3}} \rho\left(\widetilde{\beta}\left(p_{1} p_{2} p_{3}\right)\right) .
\end{aligned}
$$

Здесь буквы $p_{1}, p_{2}$ и $p_{3}$ обозначают простые числа. Поскольку количество простых чисел, не превосходящих $x$, имеет асимптотику $x / \ln x$, используя преобразование Абеля, от сумм по простым числам можно перейти к суммам по произвольным натуральным числам:

$$
\begin{aligned}
& \Psi_{2}\left(x, x^{\beta_{0}}, x^{\alpha_{1}}, x^{\beta_{1}}, x^{\alpha_{2}}, x^{\beta_{2}}\right) \approx \\
& \quad \sum_{x^{\alpha_{1}} \leqslant k_{1}<x^{\beta_{1}} \max \left\{k_{1}, x^{\alpha}\right\} \leqslant k_{2}<x^{\beta_{2}}} \frac{x}{\left(k_{1} \ln k_{1}\right)\left(k_{2} \ln k_{2}\right)} \rho\left(\widetilde{\beta}\left(k_{1} k_{2}\right)\right) \\
& \Psi_{3}\left(x, x^{\beta_{0}}, x^{\alpha_{1}}, x^{\beta_{1}}, x^{\alpha_{2}}, x^{\beta_{2}}\right) \approx \\
& \quad \sum_{x^{\alpha_{1} \leqslant k_{1}<x^{\beta_{1}} \max \left\{k_{1}, x^{\alpha_{2}}\right\} \leqslant k_{2}<x^{\beta_{2}}}} \sum_{k_{2} \leqslant k_{3}<x^{\beta_{2}}} \frac{x \rho\left(\widetilde{\beta}\left(k_{1} k_{2} k_{3}\right)\right.}{\left(k_{1} \ln k_{1}\right)\left(k_{2} \ln k_{2}\right)\left(k_{3} \ln k_{3}\right)} .
\end{aligned}
$$


Заменяя кратные суммы интегралами, найдем, что

и

$$
\begin{aligned}
& \Psi_{2}\left(x, x^{\beta_{0}}, x^{\alpha_{1}}, x^{\beta_{1}}, x^{\alpha_{2}}, x^{\beta_{2}}\right) \approx \\
& \quad \approx x \int_{x^{\alpha_{1}}}^{x^{\beta_{1}}} \int_{\max \left\{t_{1}, x^{\alpha_{2}}\right\}}^{x^{\beta_{2}}} \rho\left(\widetilde{\beta}\left(t_{1} t_{2}\right)\right) \frac{d t_{1}}{t_{1} \ln t_{1}} \frac{d t_{2}}{t_{2} \ln t_{2}}
\end{aligned}
$$

$$
\begin{aligned}
& \Psi_{3}\left(x, x^{\beta_{0}}, x^{\alpha_{1}}, x^{\beta_{1}}, x^{\alpha_{2}}, x^{\beta_{2}}\right) \approx \\
& \quad \approx x \int_{x^{\alpha_{1}}}^{x^{\beta_{1}}} \int_{\max \left\{t_{1}, x^{\alpha_{2}}\right\}}^{x^{\beta_{2}}} \int_{t_{2}}^{x^{\beta_{2}}} \rho\left(\widetilde{\beta}\left(t_{1} t_{2} t_{3}\right)\right) \frac{d t_{1}}{t_{1} \ln t_{1}} \frac{d t_{2}}{t_{2} \ln t_{2}} \frac{d t_{3}}{t_{3} \ln t_{3}} .
\end{aligned}
$$

Сделаем в интегралах замену переменных $\lambda_{i}=\left(\ln t_{i}\right) /(\ln x), i=1,2,3$. Тогда получим, что

$$
\begin{aligned}
& \Psi_{2}\left(x, x^{\beta_{0}}, x^{\alpha_{1}}, x^{\beta_{1}}, x^{\alpha_{2}}, x^{\beta_{2}}\right) \approx \\
& \quad \approx x \int_{\alpha_{1}}^{\beta_{1}} \int_{\max \left\{\lambda_{1}, \alpha_{2}\right\}}^{\beta_{2}} \rho\left(\frac{1-\lambda_{1}-\lambda_{2}}{\beta_{0}}\right) \frac{d \lambda_{1}}{\lambda_{1}} \frac{d \lambda_{2}}{\lambda_{2}}
\end{aligned}
$$

и

$$
\begin{aligned}
& \Psi_{3}\left(x, x^{\beta_{0}}, x^{\alpha_{1}}, x^{\beta_{1}}, x^{\alpha_{2}}, x^{\beta_{2}}\right) \approx \\
& \quad \approx x \int_{\alpha_{1}}^{\beta_{1}} \int_{\max \left\{\lambda_{1}, \alpha_{2}\right\}}^{\beta_{2}} \int_{\lambda_{2}}^{\beta_{2}} \rho\left(\frac{1-\lambda_{1}-\lambda_{2}-\lambda_{3}}{\beta_{0}}\right) \frac{d \lambda_{1}}{\lambda_{1}} \frac{d \lambda_{2}}{\lambda_{2}} \frac{d \lambda_{3}}{\lambda_{3}},
\end{aligned}
$$

и, значит, по определению функций $G_{2}$ и $G_{3}$

$$
\begin{aligned}
& G_{2}\left(\beta_{0}, \alpha_{1}, \beta_{1}, \alpha_{2}, \beta_{2}\right)= \\
& \quad=\int_{\alpha_{1}}^{\beta_{1}} \int_{\max \left\{\lambda_{1}, \alpha_{2}\right\}}^{\beta_{2}} \rho\left(\frac{1-\lambda_{1}-\lambda_{2}}{\beta_{0}}\right) \frac{d \lambda_{1}}{\lambda_{1}} \frac{d \lambda_{2}}{\lambda_{2}}
\end{aligned}
$$

и

$$
\begin{aligned}
& G_{3}\left(\beta_{0}, \alpha_{1}, \beta_{1}, \alpha_{2}, \beta_{2}\right)= \\
& \quad=\int_{\alpha_{1}}^{\beta_{1}} \int_{\max \left\{\lambda_{1}, \alpha_{2}\right\}}^{\beta_{2}} \int_{\lambda_{2}}^{\beta_{2}} \rho\left(\frac{1-\lambda_{1}-\lambda_{2}-\lambda_{3}}{\beta_{0}}\right) \frac{d \lambda_{1}}{\lambda_{1}} \frac{d \lambda_{2}}{\lambda_{2}} \frac{d \lambda_{3}}{\lambda_{3}} .
\end{aligned}
$$

Заметим, что интеграл $G_{3}$ можно записать в виде

$$
\begin{aligned}
& G_{3}\left(\beta_{0}, \alpha_{1}, \beta_{1}, \alpha_{2}, \beta_{2}\right)= \\
& \quad=\int_{\alpha_{1}}^{\beta_{1}} \iint_{\substack{\left(\lambda_{2}, \lambda_{3}\right) \in\left[\max \\
\lambda_{2} \leqslant \lambda_{3}\right.}} \rho\left(\frac{\left.\left.1-\lambda_{1}, \alpha_{2}\right\} ; \beta_{2}\right]^{2}}{\beta_{0}-\lambda_{3}}\right) \frac{d \lambda_{1}}{\lambda_{1}} \frac{d \lambda_{2}}{\lambda_{2}} \frac{d \lambda_{3}}{\lambda_{3}} .
\end{aligned}
$$


Поскольку подынтегральная функция симметрична относительно перестановки переменных $\lambda_{2}$ и $\lambda_{3}$,

$$
\begin{aligned}
& G_{3}\left(\beta_{0}, \alpha_{1}, \beta_{1}, \alpha_{2}, \beta_{2}\right)= \\
& \quad=\frac{1}{2} \int_{\alpha_{1}}^{\beta_{1}} \int_{\max \left\{\lambda_{1}, \alpha_{2}\right\}}^{\beta_{2}} \int_{\max \left\{\lambda_{1}, \alpha_{2}\right\}}^{\beta_{2}} \rho\left(\frac{1-\lambda_{1}-\lambda_{2}-\lambda_{3}}{\beta_{0}}\right) \frac{d \lambda_{1}}{\lambda_{1}} \frac{d \lambda_{2}}{\lambda_{2}} \frac{d \lambda_{3}}{\lambda_{3}} .
\end{aligned}
$$

Для дальнейшего изложения потребуются интегралы

$$
\begin{aligned}
& G_{m}^{(1)}\left(\beta_{0}, \alpha_{1}, \beta_{1}, \alpha_{2}, \beta_{2}\right)= \\
& =\frac{1}{(m-1) !} \int_{\alpha_{1}}^{\beta_{1}} \int_{\alpha_{2}}^{\beta_{2}} \cdots \int_{\alpha_{2}}^{\beta_{2}} \rho\left(\frac{1-\lambda_{1}-\lambda_{2}-\cdots-\lambda_{m}}{\beta_{0}}\right) \frac{d \lambda_{1}}{\lambda_{1}} \frac{d \lambda_{2}}{\lambda_{2}} \cdots \frac{d \lambda_{m}}{\lambda_{m}}
\end{aligned}
$$

И

$$
\begin{aligned}
& G_{m}^{(2)}\left(\beta_{0}, \alpha_{1}, \beta_{1}, \beta_{2}\right)= \\
& =\frac{1}{(m-1) !} \int_{\alpha_{1}}^{\beta_{1}} \int_{\lambda_{1}}^{\beta_{2}} \cdots \int_{\lambda_{1}}^{\beta_{2}} \rho\left(\frac{1-\lambda_{1}-\lambda_{2}-\cdots-\lambda_{m}}{\beta_{0}}\right) \frac{d \lambda_{1}}{\lambda_{1}} \frac{d \lambda_{2}}{\lambda_{2}} \cdots \frac{d \lambda_{m}}{\lambda_{m}},
\end{aligned}
$$

$m=1,2, \ldots$ Очевидно, что интегралы $G_{m}$ можно выразить через функции $G_{m}^{(1)}$ и $G_{m}^{(2)}$. Точнее, имеет место следующая теорема.

Теорема 1. Пусть $m=2$ или 3. Тогда:

1) если $\alpha_{2} \geqslant \beta_{1}$, то

$$
G_{m}\left(\beta_{0}, \alpha_{1}, \beta_{1}, \alpha_{2}, \beta_{2}\right)=G_{m}^{(1)}\left(\beta_{0}, \alpha_{1}, \beta_{1}, \alpha_{2}, \beta_{2}\right),
$$

2) если $\alpha_{2}<\beta_{1}$, то

$$
G_{m}\left(\beta_{0}, \alpha_{1}, \beta_{1}, \alpha_{2}, \beta_{2}\right)=G_{m}^{(1)}\left(\beta_{0}, \alpha_{1}, \alpha_{2}, \alpha_{2}, \beta_{2}\right)+G_{m}^{(2)}\left(\beta_{0}, \alpha_{2}, \beta_{1}, \beta_{2}\right) .
$$

Таким образом, исследование функций $G_{m}$ сведено к исследованию функций $G_{m}^{(1)}$ и $G_{m}^{(2)}$.

\section{3. Леммы о преобразованиях интегралов}

Для дальнейшего преобразования интегралов $G_{m}^{(r)}$ потребуется ряд вспомогательных утверждений. 
Лемма 1. Пусть $\alpha_{1}, \beta_{1}\left(\alpha_{1}<\beta_{1}\right)$ - вещественные числа, $l_{1}(x), l_{2}(x)-\partial в е$ такие непрерывные обратимые функции, что $l_{1}(x)<l_{2}(x)$ при $x \in\left(\alpha_{1}, \beta_{1}\right)$ (см. рисунок); $\varphi(u, v)$ - интегрируемая функиия. Тогда имеет место интегральная формула

$$
\begin{aligned}
& \int_{\alpha_{1}}^{\beta_{1}} d \mu_{1} \int_{l_{1}\left(\mu_{1}\right)}^{l_{2}\left(\mu_{1}\right)} \varphi\left(\mu_{1}, \mu_{2}\right) d \mu_{2}= \\
& \quad=\int_{l_{1}\left(\alpha_{1}\right)}^{l_{1}\left(\beta_{1}\right)} d \mu_{2} \int_{\alpha_{1}}^{l_{1}^{-1}\left(\mu_{2}\right)} \varphi\left(\mu_{1}, \mu_{2}\right) d \mu_{1}+ \\
& \quad+\int_{l_{1}\left(\beta_{1}\right)}^{l_{2}\left(\alpha_{1}\right)} d \mu_{2} \int_{\alpha_{1}}^{\beta_{1}} \varphi\left(\mu_{1}, \mu_{2}\right) d \mu_{1}+\int_{l_{2}\left(\alpha_{1}\right)}^{l_{2}\left(\beta_{1}\right)} d \mu_{2} \int_{l_{2}^{-1}\left(\mu_{1}\right)}^{\beta_{1}} \varphi\left(\mu_{1}, \mu_{2}\right) d \mu_{1} .
\end{aligned}
$$

Доказательство. Возможны два случая: $l_{1}\left(\beta_{1}\right) \leqslant l_{2}\left(\alpha_{1}\right)$ (левая часть рисунка) и $l_{1}\left(\beta_{1}\right)>l_{2}\left(\alpha_{1}\right)$ (правая часть рисунка).
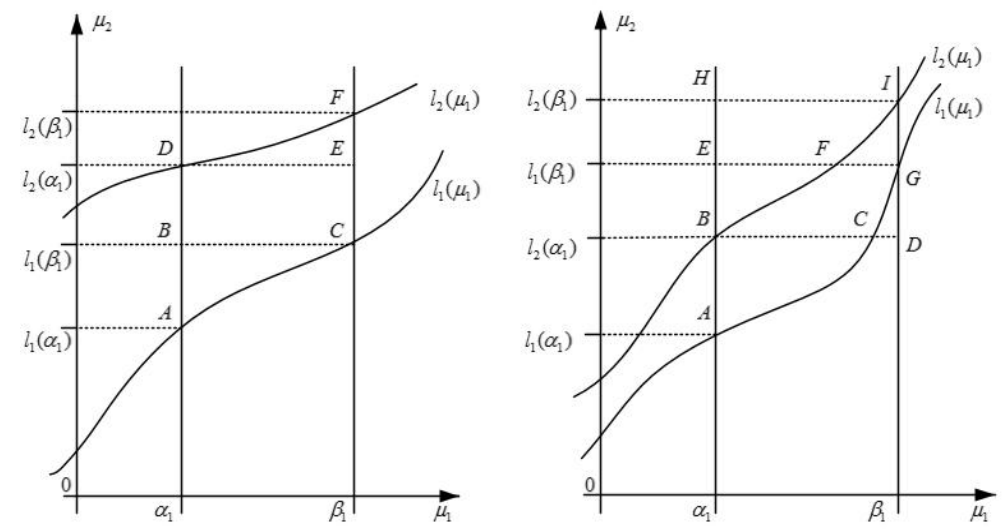

Рисунок. Графики функций к лемме 1

Рассмотрим каждый из этих случаев по отдельности.

Пусть $l_{1}\left(\beta_{1}\right) \leqslant l_{2}\left(\alpha_{1}\right)$. Тогда интеграл в левой части равенства (1) равен двумерному интегралу от функции $\varphi$ по криволинейной трапеции $A D F C$ (см. левую часть рисунка). Эту трапецию можно представить в виде объединения прямоугольника $B D E C$ и двух криволинейных треугольников: $A B C$ и $D F E$. Таким образом, исходный интеграл представляется в виде суммы трех интегралов. Эти интегралы как раз и выписаны в правой части равенства (1): первый и третий интегралы правой части равны соответственно интегралам по криволинейным треугольникам $A B C$ и $D F E$, а второй интеграл равен интегралу по прямоугольнику $B D E C$. Это доказывает равенство (1) в первом случае. 
Пусть $l_{1}\left(\beta_{1}\right)>l_{2}\left(\alpha_{1}\right)$. Тогда интеграл в левой части равенства (1) равен двумерному интегралу от функции $\varphi$ по криволинейной трапеции $A B I G$ (см. правую часть рисунка).

В правой части равенства (1) выписана сумма трех интегралов: первый и третий интегралы берутся по криволинейным треугольникам $A E G$ и $B I D$ соответственно, а второй интеграл равен интегралу по прямоугольнику $B E G D$, взятому с обратным знаком.

Интеграл по криволинейному треугольнику $A E G$ равен сумме трех интегралов: интеграла по криволинейному треугольнику $A B C$ (обозначим его $I_{1}$ ), интеграла по криволинейному треугольнику $B E F$ (обозначим его $I_{2}$ ) и интеграла по криволинейной трапеции $B F G C$ (обозначим его $I_{3}$ ).

Аналогичным образом интеграл по криволинейному треугольнику $B I D$ представим в виде суммы трех интегралов: интеграла по криволинейному треугольнику $F I G$ (обозначим его $I_{4}$ ), интеграла по криволинейному треугольнику $C G D$ (обозначим его $I_{5}$ ) и интеграла $I_{3}$ по криволинейной трапеции $B F G C$.

Сумма интегралов $I_{2}+I_{3}+I_{5}$ равна интегралу по прямоугольнику $B E G D$, этот интеграл в правой части формулы (1) вычитается. Следовательно, в итоге правая часть формулы (1) равна сумме интегралов $I_{1}+I_{3}+I_{4}$, т. е. как раз левой части. Это доказывает равенство (1) во втором случае.

Лемма 2. В лемме 1 можно снять условие $\alpha_{1}<\beta_{1}$.

Доказательство. При $\alpha_{1}=\beta_{1}$ левая часть в формуле (1) равна нулю. Правая часть непрерывна по параметру $\alpha_{1}$, поэтому при $\alpha_{1}=\beta_{1}$ она тоже равна нулю и равенство выполнено. Пусть $\alpha_{1}>\beta_{1}$. Введем обозначения $\bar{\alpha}_{1}=\beta_{1}$, $\bar{\beta}_{1}=\alpha_{1}$.

Применяя к параметрам $\bar{\alpha}_{1}<\bar{\beta}_{1}$ лемму 1 , получаем равенство

$$
\begin{aligned}
& \int_{\bar{\alpha}_{1}}^{\bar{\beta}_{1}} d \mu_{1} \int_{l_{1}\left(\mu_{1}\right)}^{l_{2}\left(\mu_{1}\right)} \varphi\left(\mu_{1}, \mu_{2}\right) d \mu_{2}= \\
& =\int_{l_{1}\left(\bar{\alpha}_{1}\right)}^{l_{1}\left(\bar{\beta}_{1}\right)} d \mu_{2} \int_{\bar{\alpha}_{1}}^{l_{1}^{-1}\left(\mu_{2}\right)} \varphi\left(\mu_{1}, \mu_{2}\right) d \mu_{1}+ \\
& \quad+\int_{l_{1}\left(\bar{\beta}_{1}\right)}^{l_{2}\left(\bar{\alpha}_{1}\right)} d \mu_{2} \int_{\bar{\alpha}_{1}}^{\bar{\beta}_{1}} \varphi\left(\mu_{1}, \mu_{2}\right) d \mu_{1}+\int_{l_{2}\left(\bar{\alpha}_{1}\right)}^{l_{2}\left(\bar{\beta}_{1}\right)} d \mu_{2} \int_{l_{2}^{-1}\left(\mu_{1}\right)}^{\bar{\beta}_{1}} \varphi\left(\mu_{1}, \mu_{2}\right) d \mu_{1},
\end{aligned}
$$


которое в исходных обозначениях имеет вид

$$
\begin{aligned}
& \int_{\beta_{1}}^{\alpha_{1}} d \mu_{1} \int_{l_{1}\left(\mu_{1}\right)}^{l_{2}\left(\mu_{1}\right)} \varphi\left(\mu_{1}, \mu_{2}\right) d \mu_{2}= \\
& =\int_{l_{1}\left(\beta_{1}\right)}^{l_{1}\left(\alpha_{1}\right)} d \mu_{2} \int_{\beta_{1}}^{l_{1}^{-1}\left(\mu_{2}\right)} \varphi\left(\mu_{1}, \mu_{2}\right) d \mu_{1}+ \\
& \quad+\int_{l_{1}\left(\alpha_{1}\right)}^{l_{2}\left(\beta_{1}\right)} d \mu_{2} \int_{\beta_{1}}^{\alpha_{1}} \varphi\left(\mu_{1}, \mu_{2}\right) d \mu_{1}+\int_{l_{2}\left(\beta_{1}\right)}^{l_{2}\left(\alpha_{1}\right)} d \mu_{2} \int_{l_{2}^{-1}\left(\mu_{1}\right)}^{\alpha_{1}} \varphi\left(\mu_{1}, \mu_{2}\right) d \mu_{1}
\end{aligned}
$$

Домножив обе части этого равенства на -1 , придем к равенству

$$
\begin{aligned}
& \int_{\alpha_{1}}^{\beta_{1}} d \mu_{1} \int_{l_{1}\left(\mu_{1}\right)}^{l_{2}\left(\mu_{1}\right)} \varphi\left(\mu_{1}, \mu_{2}\right) d \mu_{2}= \\
& =\int_{l_{1}\left(\alpha_{1}\right)}^{l_{1}\left(\beta_{1}\right)} d \mu_{2} \int_{\beta_{1}}^{l_{1}^{-1}\left(\mu_{2}\right)} \varphi\left(\mu_{1}, \mu_{2}\right) d \mu_{1}+ \\
& \quad+\int_{l_{1}\left(\alpha_{1}\right)}^{l_{2}\left(\beta_{1}\right)} d \mu_{2} \int_{\alpha_{1}}^{\beta_{1}} \varphi\left(\mu_{1}, \mu_{2}\right) d \mu_{1}+\int_{l_{2}\left(\alpha_{1}\right)}^{l_{2}\left(\beta_{1}\right)} d \mu_{2} \int_{l_{2}^{-1}\left(\mu_{1}\right)}^{\alpha_{1}} \varphi\left(\mu_{1}, \mu_{2}\right) d \mu_{1} .
\end{aligned}
$$

Левая часть формулы (2) совпадает с левой частью формулы (1). Для доказательства леммы осталось показать, что и правые части этих формул тоже совпадают. Для этого преобразуем правую часть формулы (2).

Правая часть состоит из трех слагаемых, которые обозначим $I_{1}, I_{2}$ и $I_{3}$. Внутренний интеграл в $I_{1}$ представим в виде суммы

$$
\int_{\beta_{1}}^{l_{1}^{-1}\left(\mu_{2}\right)} \varphi\left(\mu_{1}, \mu_{2}\right) d \mu_{1}=\int_{\beta_{1}}^{\alpha_{1}} \varphi\left(\mu_{1}, \mu_{2}\right) d \mu_{1}+\int_{\alpha_{1}}^{l_{1}^{-1}\left(\mu_{2}\right)} \varphi\left(\mu_{1}, \mu_{2}\right) d \mu_{1}=J+J_{1} .
$$

Аналогичным образом представим в виде суммы внутренний интеграл в $I_{3}$ :

$$
\int_{l_{2}^{-1}\left(\mu_{1}\right)}^{\alpha_{1}} \varphi\left(\mu_{1}, \mu_{2}\right) d \mu_{1}=\int_{l_{2}^{-1}\left(\mu_{1}\right)}^{\beta_{1}} \varphi\left(\mu_{1}, \mu_{2}\right) d \mu_{1}+\int_{\beta_{1}}^{\alpha_{1}} \varphi\left(\mu_{1}, \mu_{2}\right) d \mu_{1}=J_{3}+J
$$

Заметим, что интегралы

$$
\int_{l_{1}\left(\alpha_{1}\right)}^{l_{1}\left(\beta_{1}\right)} J_{1} d \mu_{2} \quad \text { и } \quad \int_{l_{2}\left(\alpha_{1}\right)}^{l_{2}\left(\beta_{1}\right)} J_{3} d \mu_{2}
$$


равны, соответственно, первому и третьему слагаемым правой части формулы (1). Поэтому для доказательства леммы остается показать, что второе слагаемое правой части равно

$$
\int_{l_{1}\left(\alpha_{1}\right)}^{l_{1}\left(\beta_{1}\right)} J d \mu_{2}+\int_{l_{2}\left(\alpha_{1}\right)}^{l_{2}\left(\beta_{1}\right)} J d \mu_{2}-\int_{l_{1}\left(\alpha_{1}\right)}^{l_{2}\left(\beta_{1}\right)} J d \mu_{2} .
$$

Переписывая последнюю сумму в виде

$$
\int_{l_{2}\left(\alpha_{1}\right)}^{l_{2}\left(\beta_{1}\right)} J d \mu_{2}+\int_{l_{2}\left(\beta_{1}\right)}^{l_{1}\left(\alpha_{1}\right)} J d \mu_{2}+\int_{l_{1}\left(\alpha_{1}\right)}^{l_{1}\left(\beta_{1}\right)} J d \mu_{2},
$$

замечаем, что она равна интегралу

$$
\int_{l_{2}\left(\alpha_{1}\right)}^{l_{1}\left(\beta_{1}\right)} J d \mu_{2}=\int_{l_{1}\left(\beta_{1}\right)}^{l_{2}\left(\alpha_{1}\right)} d \mu_{2} \int_{\alpha_{1}}^{\beta_{1}} \varphi\left(\mu_{1}, \mu_{2}\right) d \mu_{1},
$$

что и требовалось.

Лемма 3. В лемме 1 можно заменить условие $l_{1}(x)<l_{2}(x)$ более слабым условием $l_{1}(x) \neq l_{2}(x)$ при $x \in\left(\alpha_{1}, \beta_{1}\right)$.

Доказательство. Допустим, что $l_{1}(x)>l_{2}(x)$ при $x \in\left(\alpha_{1}, \beta_{1}\right)$. Введем обозначения: $\bar{l}_{1}(x)=l_{2}(x), \bar{l}_{2}(x)=l_{1}(x)$.

Применяя к функциям $\bar{l}_{1}(x)<\bar{l}_{2}(x)$ лемму 2 , получаем равенство

$$
\begin{aligned}
& \int_{\alpha_{1}}^{\beta_{1}} d \mu_{1} \int_{\bar{l}_{1}\left(\mu_{1}\right)}^{\bar{l}_{2}\left(\mu_{1}\right)} \varphi\left(\mu_{1}, \mu_{2}\right) d \mu_{2}= \\
& =\int_{\bar{l}_{1}\left(\alpha_{1}\right)}^{\bar{l}_{1}\left(\beta_{1}\right)} d \mu_{2} \int_{\alpha_{1}}^{\bar{l}_{1}^{-1}\left(\mu_{2}\right)} \varphi\left(\mu_{1}, \mu_{2}\right) d \mu_{1}+ \\
& \quad+\int_{\bar{l}_{1}\left(\beta_{1}\right)}^{\bar{l}_{2}\left(\alpha_{1}\right)} d \mu_{2} \int_{\alpha_{1}}^{\beta_{1}} \varphi\left(\mu_{1}, \mu_{2}\right) d \mu_{1}+\int_{\bar{l}_{2}\left(\alpha_{1}\right)}^{\bar{l}_{2}\left(\beta_{1}\right)} d \mu_{2} \int_{\bar{l}_{2}^{-1}\left(\mu_{1}\right)}^{\beta_{1}} \varphi\left(\mu_{1}, \mu_{2}\right) d \mu_{1},
\end{aligned}
$$

которое в исходных обозначениях имеет вид

$$
\begin{aligned}
& \int_{\alpha_{1}}^{\beta_{1}} d \mu_{1} \int_{l_{2}\left(\mu_{1}\right)}^{l_{1}\left(\mu_{1}\right)} \varphi\left(\mu_{1}, \mu_{2}\right) d \mu_{2}= \\
& =\int_{l_{2}\left(\alpha_{1}\right)}^{l_{2}\left(\beta_{1}\right)} d \mu_{2} \int_{\alpha_{1}}^{l_{2}^{-1}\left(\mu_{2}\right)} \varphi\left(\mu_{1}, \mu_{2}\right) d \mu_{1}+ \\
& \quad+\int_{l_{2}\left(\beta_{1}\right)}^{l_{1}\left(\alpha_{1}\right)} d \mu_{2} \int_{\alpha_{1}}^{\beta_{1}} \varphi\left(\mu_{1}, \mu_{2}\right) d \mu_{1}+\int_{l_{1}\left(\alpha_{1}\right)}^{l_{1}\left(\beta_{1}\right)} d \mu_{2} \int_{l_{1}^{-1}\left(\mu_{1}\right)}^{\beta_{1}} \varphi\left(\mu_{1}, \mu_{2}\right) d \mu_{1} .
\end{aligned}
$$


Домножив обе части этого равенства на -1, придем к равенству

$$
\begin{aligned}
& \int_{\alpha_{1}}^{\beta_{1}} d \mu_{1} \int_{l_{1}\left(\mu_{1}\right)}^{l_{2}\left(\mu_{1}\right)} \varphi\left(\mu_{1}, \mu_{2}\right) d \mu_{2}= \\
& =\int_{l_{2}\left(\alpha_{1}\right)}^{l_{2}\left(\beta_{1}\right)} d \mu_{2} \int_{l_{2}^{-1}\left(\mu_{2}\right)}^{\alpha_{1}} \varphi\left(\mu_{1}, \mu_{2}\right) d \mu_{1}+ \\
& \quad \int_{l_{2}\left(\beta_{1}\right)}^{l_{1}\left(\alpha_{1}\right)} d \mu_{2} \int_{\beta_{1}}^{\alpha_{1}} \varphi\left(\mu_{1}, \mu_{2}\right) d \mu_{1}+\int_{l_{1}\left(\alpha_{1}\right)}^{l_{1}\left(\beta_{1}\right)} d \mu_{2} \int_{\beta_{1}}^{l_{1}^{-1}\left(\mu_{1}\right)} \varphi\left(\mu_{1}, \mu_{2}\right) d \mu_{1} .
\end{aligned}
$$

Левая часть формулы (3) совпадает с левой частью формулы (1). Для доказательства леммы осталось показать, что и правые части этих формул тоже совпадают. Для этого преобразуем правую часть формулы (3).

Правая часть состоит из трех слагаемых, которые обозначим $I_{3}, I_{2}$ и $I_{1}$. Внутренний интеграл в $I_{3}$ представим в виде суммы

$$
\begin{aligned}
& \int_{l_{2}^{-1}\left(\mu_{2}\right)}^{\alpha_{1}} \varphi\left(\mu_{1}, \mu_{2}\right) d \mu_{1}= \\
& =\int_{l_{2}^{-1}\left(\mu_{2}\right)}^{\beta_{1}} \varphi\left(\mu_{1}, \mu_{2}\right) d \mu_{1}+\int_{\beta_{1}}^{\alpha_{1}} \varphi\left(\mu_{1}, \mu_{2}\right) d \mu_{1}=J_{3}+J
\end{aligned}
$$

Аналогичным образом внутренний интеграл в $I_{1}$ представим в виде суммы

$$
\begin{aligned}
& \int_{\beta_{1}}^{l_{1}^{-1}\left(\mu_{1}\right)} \varphi\left(\mu_{1}, \mu_{2}\right) d \mu_{1}= \\
& =\int_{\beta_{1}}^{\alpha_{1}} \varphi\left(\mu_{1}, \mu_{2}\right) d \mu_{1}+\int_{\alpha_{1}}^{l_{1}^{-1}\left(\mu_{1}\right)} \varphi\left(\mu_{1}, \mu_{2}\right) d \mu_{1}=J+J_{1} .
\end{aligned}
$$

Заметим, что интегралы

$$
\int_{l_{1}\left(\alpha_{1}\right)}^{l_{1}\left(\beta_{1}\right)} J_{1} d \mu_{2} \quad \text { и } \quad \int_{l_{2}\left(\alpha_{1}\right)}^{l_{2}\left(\beta_{1}\right)} J_{3} d \mu_{2}
$$

равны, соответственно, первому и третьему слагаемым правой части формулы (1). Поэтому для доказательства леммы остается показать, что второе слагаемое правой части равно

$$
\int_{l_{1}\left(\alpha_{1}\right)}^{l_{1}\left(\beta_{1}\right)} J d \mu_{2}+\int_{l_{2}\left(\alpha_{1}\right)}^{l_{2}\left(\beta_{1}\right)} J d \mu_{2}+\int_{l_{2}\left(\beta_{1}\right)}^{l_{1}\left(\alpha_{1}\right)} J d \mu_{2} .
$$


Переписывая последнюю сумму в виде

$$
\int_{l_{2}\left(\alpha_{1}\right)}^{l_{2}\left(\beta_{1}\right)} J d \mu_{2}+\int_{l_{2}\left(\beta_{1}\right)}^{l_{1}\left(\alpha_{1}\right)} J d \mu_{2}+\int_{l_{1}\left(\alpha_{1}\right)}^{l_{1}\left(\beta_{1}\right)} J d \mu_{2},
$$

замечаем, что она равна интегралу

$$
\int_{l_{2}\left(\alpha_{1}\right)}^{l_{1}\left(\beta_{1}\right)} J d \mu_{2}=\int_{l_{1}\left(\beta_{1}\right)}^{l_{2}\left(\alpha_{1}\right)} d \mu_{2} \int_{\alpha_{1}}^{\beta_{1}} \varphi\left(\mu_{1}, \mu_{2}\right) d \mu_{1},
$$

что и требовалось доказать.

Лемма 4. Пусть $\alpha_{1}, \beta_{1}, \alpha_{2}, \beta_{2}, p \neq 0$ u $q \neq 0$ - такие вещественные числа, что $\alpha_{2}+p \mu_{1} \neq \beta_{2}+q \mu_{1}$ при $\mu_{1} \in\left(\alpha_{1}, \beta_{1}\right)$, а $\varphi(u, v)-$ интегрируемая функиия. Тогда имеет место интегральная формула

$$
\begin{aligned}
& \int_{\alpha_{1}}^{\beta_{1}} d \mu_{1} \int_{\alpha_{2}+p \mu_{1}}^{\beta_{2}+q \mu_{1}} \varphi\left(\mu_{1}, \mu_{2}\right) d \mu_{2}= \\
& =\sum_{u, v} e(u, v) \int_{u+\alpha_{1}}^{v+\beta_{1}} d \mu_{2} \int_{l_{u}\left(\mu_{2}\right)}^{l_{v}\left(\mu_{2}\right)} \varphi\left(\mu_{1}, \mu_{2}\right) d \mu_{1},
\end{aligned}
$$

где

$$
\begin{aligned}
& e(u, v)=\left\{\begin{array}{rrr}
-1 & \text { при } & (u, v)=\left(\alpha_{2}+(p-1) \alpha_{1}, \alpha_{2}+(p-1) \beta_{1}\right), \\
+1 & n р u & (u, v)=\left(\beta_{2}+(q-1) \alpha_{1}, \alpha_{2}+(p-1) \beta_{1}\right), \\
-1 & n p u & (u, v)=\left(\beta_{2}+(q-1) \alpha_{1}, \beta_{2}+(q-1) \beta_{1}\right), \\
0 & \text { в остальных случаях }
\end{array}\right. \\
& l_{u}\left(\mu_{2}\right)=\left\{\begin{array}{ccc}
\frac{\mu_{2}-\alpha_{2}}{p} & n р и & u=\alpha_{2}+(p-1) \alpha_{1}, \\
\beta_{1} & n р и & u=\beta_{2}+(q-1) \alpha_{1}
\end{array}\right. \\
& l_{v}\left(\mu_{2}\right)=\left\{\begin{array}{ccc}
\alpha_{1} & \text { nри } & v=\alpha_{2}+(p-1) \beta_{1}, \\
\frac{\mu_{2}-\beta_{2}}{q} & \text { nри } & v=\beta_{2}+(q-1) \beta_{1} .
\end{array}\right.
\end{aligned}
$$


Доказательство. Пользуясь леммами 1-3, выпишем формулу

$$
\begin{aligned}
& \int_{\alpha_{1}}^{\beta_{1}} d \mu_{1} \int_{\alpha_{2}+p \mu_{1}}^{\beta_{2}+q \mu_{1}} \varphi\left(\mu_{1}, \mu_{2}\right) d \mu_{2}= \\
& =\int_{\alpha_{2}+p \alpha_{1}}^{\alpha_{2}+p \beta_{1}} d \mu_{2} \int_{\alpha_{1}}^{\left(\mu_{2}-\alpha_{2}\right) / p} \varphi\left(\mu_{1}, \mu_{2}\right) d \mu_{1}+ \\
& +\int_{\alpha_{2}+p \beta_{1}}^{\beta_{2}+q \alpha_{1}} d \mu_{2} \int_{\alpha_{1}}^{\beta_{1}} \varphi\left(\mu_{1}, \mu_{2}\right) d \mu_{1}+\int_{\beta_{2}+q \alpha_{1}}^{\beta_{2}+q \beta_{1}} d \mu_{2} \int_{\left(\mu_{2}-\beta_{2}\right) / q}^{\beta_{1}} \varphi\left(\mu_{1}, \mu_{2}\right) d \mu_{1} .
\end{aligned}
$$

Проверим, что в правых частях формул (4) и (5) стоит одна и та же величина.

Если взять

$$
(u, v)=\left(\alpha_{2}+(p-1) \alpha_{1}, \alpha_{2}+(p-1) \beta_{1}\right),
$$

то соответствующее слагаемое суммы из правой части формулы (4) станет равным

$$
-\int_{\alpha_{2}+p \alpha_{1}}^{\alpha_{2}+p \beta_{1}} d \mu_{2} \int_{\left(\mu_{2}-\alpha_{2}\right) / p}^{\alpha_{1}} \varphi\left(\mu_{1}, \mu_{2}\right) d \mu_{1},
$$

т. е. оно будет совпадать с первым слагаемым суммы из правой части формулы (5).

Если взять

$$
(u, v)=\left(\beta_{2}+(q-1) \alpha_{1}, \beta_{2}+(q-1) \beta_{1}\right),
$$

то соответствующее слагаемое суммы из правой части формулы (4) станет равным

$$
-\int_{\beta_{2}+q \alpha_{1}}^{\beta_{2}+q \beta_{1}} d \mu_{2} \int_{\beta_{1}}^{\left(\mu_{2}-\beta_{2}\right) / q} \varphi\left(\mu_{1}, \mu_{2}\right) d \mu_{1}
$$

т. е. оно будет совпадать с третьим слагаемым суммы из правой части формулы (5).

Если взять

$$
(u, v)=\left(\beta_{2}+(q-1) \alpha_{1}, \alpha_{2}+(p-1) \beta_{1}\right),
$$

то соответствующее слагаемое суммы из правой части формулы (4) станет равным

$$
\int_{\beta_{2}+q \alpha_{1}}^{\alpha_{2}+p \beta_{1}} d \mu_{2} \int_{\beta_{1}}^{\alpha_{1}} \varphi\left(\mu_{1}, \mu_{2}\right) d \mu_{1}
$$

т. е. оно будет совпадать со вторым слагаемым суммы из правой части формулы (5).

Остальные слагаемые суммы правой части формулы (4) равны нулю, что и доказывает лемму. 
Лемма 5. При выполнении условий леммы 4 имеет место формула

$$
\int_{l_{u}\left(\mu_{2}\right)}^{l_{v}\left(\mu_{2}\right)} \frac{d \mu_{1}}{\mu_{1}\left(\mu_{2}-\mu_{1}\right)}=\frac{1}{\mu_{2}} \sum_{i=0}^{2} \varepsilon_{i}(u, v) \ln \frac{\mu_{2}+\theta_{i} \gamma_{i}(u, v)}{\gamma_{i}(u, v)}
$$

где

$$
\begin{aligned}
& \gamma_{0}(u, v)=\left\{\begin{array}{lll}
\alpha_{2} & \text { nрu } & u=\alpha_{2}+(p-1) \alpha_{1}, \\
\beta_{1} & \text { nрu } & u=\beta_{2}+(q-1) \alpha_{1},
\end{array}\right. \\
& \gamma_{1}(u, v)=\left\{\begin{array}{lll}
\alpha_{1} & \text { nрu } & v=\alpha_{2}+(p-1) \beta_{1}, \\
\beta_{2} & \text { nрu } & v=\beta_{2}+(q-1) \beta_{1}
\end{array}\right. \\
& \gamma_{2}(u, v)=\left\{\begin{array}{llll}
\frac{\alpha_{2}}{p-1} & n p u & (u, v)=\left(\alpha_{2}+(p-1) \alpha_{1}, \alpha_{2}+(p-1) \beta_{1}\right), & p \neq 1, \\
\frac{\beta_{2}}{q-1} & n p u & (u, v)=\left(\beta_{2}+(q-1) \alpha_{1}, \beta_{2}+(q-1) \beta_{1}\right), & q \neq 1,
\end{array}\right. \\
& \varepsilon_{0}(u, v)=\left\{\begin{array}{rcr}
-1 & \text { при } & u=\alpha_{2}+(p-1) \alpha_{1} \\
+1 & \text { при } & u=\beta_{2}+(q-1) \alpha_{1} \\
0 & \text { в остальных случаях }
\end{array}\right.
\end{aligned}
$$

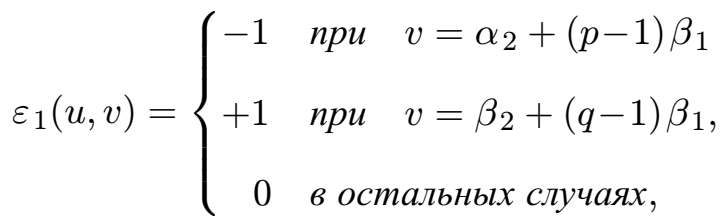

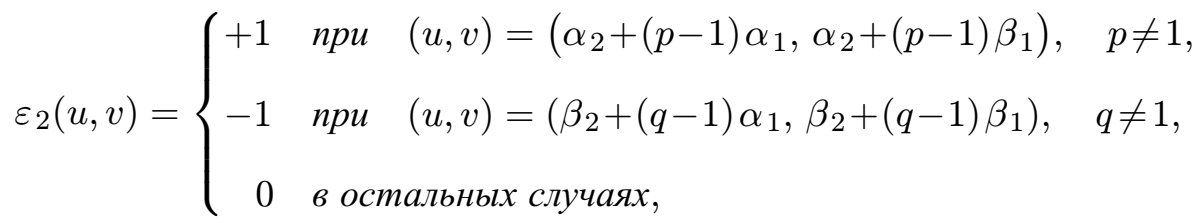

$$
\begin{aligned}
& \theta_{i}= \begin{cases}-1 & \text { при } i<2 \\
+1 & \text { в противном случае. }\end{cases}
\end{aligned}
$$


Доказательство. Легко подсчитать, что указанный в формулировке леммы интеграл равен

$$
\left.\frac{1}{\mu_{2}} \ln \frac{\mu_{1}}{\mu_{2}-\mu_{1}}\right|_{\mu_{1}=l_{u}\left(\mu_{2}\right)} ^{\mu_{1}=l_{v}\left(\mu_{2}\right)}=\frac{1}{\mu_{2}} \ln \frac{l_{v}\left(\mu_{2}\right)\left(\mu_{2}-l_{u}\left(\mu_{2}\right)\right)}{\left(\mu_{2}-l_{v}\left(\mu_{2}\right)\right) l_{u}\left(\mu_{2}\right)} .
$$

Поэтому в случае

$$
(u, v)=\left(\alpha_{2}+(p-1) \alpha_{1}, \alpha_{2}+(p-1) \beta_{1}\right)
$$

этот интеграл равен

$$
\frac{1}{\mu_{2}} \ln \frac{\alpha_{1}\left(\mu_{2}-\left(\mu_{2}-\alpha_{2}\right) / p\right)}{\left(\mu_{2}-\alpha_{1}\right)\left(\mu_{2}-\alpha_{2}\right) / p}=\frac{1}{\mu_{2}} \ln \left(\frac{\alpha_{1}}{\mu_{2}-\alpha_{1}} \frac{\alpha_{2}}{\mu_{2}-\alpha_{2}} \frac{(p-1) \mu_{2}+\alpha_{2}}{\alpha_{2}}\right),
$$

в случае

$$
(u, v)=\left(\beta_{2}+(q-1) \alpha_{1}, \alpha_{2}+(p-1) \beta_{1}\right)
$$

он равен

$$
\frac{1}{\mu_{2}} \ln \frac{\alpha_{1}\left(\mu_{2}-\beta_{1}\right)}{\left(\mu_{2}-\alpha_{1}\right) \beta_{1}}
$$

а в случае

$$
(u, v)=\left(\beta_{2}+(q-1) \alpha_{1}, \beta_{2}+(q-1) \beta_{1}\right)
$$

он равен

$\frac{1}{\mu_{2}} \ln \frac{\left(\left(\mu_{2}-\beta_{2}\right) / q\right)\left(\mu_{2}-\beta_{1}\right)}{\left(\mu_{2}-\left(\mu_{2}-\beta_{2}\right) / q\right) \beta_{1}}=\frac{1}{\mu_{2}} \ln \left(\frac{\mu_{2}-\beta_{2}}{\beta_{2}} \frac{\beta_{2}}{(q-1) \mu_{2}+\beta_{2}} \frac{\mu_{2}-\beta_{1}}{\beta_{1}}\right)$.

В первом случае имеем $\gamma_{0}=\alpha_{2}, \gamma_{1}=\alpha_{1}, \varepsilon_{0}=-1, \varepsilon_{1}=-1$,

$$
\left(\gamma_{2}, \varepsilon_{2}\right)=\left(\frac{\alpha_{2}}{p-1}, 1\right)
$$

при $p \neq 1$ и $\varepsilon_{2}=0$ при $p=1$.

Во втором случае имеем $\gamma_{0}=\beta_{0}, \gamma_{1}=\alpha_{1}, \varepsilon_{0}=1, \varepsilon_{1}=-1, \varepsilon_{2}=0$.

В третьем случае имеем $\gamma_{0}=\beta_{1}, \gamma_{1}=\beta_{2}, \varepsilon_{0}=1, \varepsilon_{1}=1$,

$$
\left(\gamma_{2}, \varepsilon_{2}\right)=\left(\frac{\beta_{2}}{q-1},-1\right)
$$

при $q \neq 1$ и $\varepsilon_{2}=0$ при $q=1$.

Очевидно, что во всех трех случаях полученное выражение равно значению приведенной в условии леммы суммы. 


\section{4. Понижение размерности интегрирования}

Рассмотрим интегралы

$$
G_{2}^{(1)}\left(\beta_{0}, \alpha_{1}, \beta_{1}, \alpha_{2}, \beta_{2}\right)=\int_{\alpha_{1}}^{\beta_{1}} \int_{\alpha_{2}}^{\beta_{2}} \rho\left(\frac{1-\lambda_{1}-\lambda_{2}}{\beta_{0}}\right) \frac{d \lambda_{1}}{\lambda_{1}} \frac{d \lambda_{2}}{\lambda_{2}}
$$

и

$$
\begin{aligned}
& G_{3}^{(1)}\left(\beta_{0}, \alpha_{1}, \beta_{1}, \beta_{2}\right)= \\
& \quad=\frac{1}{2} \int_{\alpha_{1}}^{\beta_{1}} \int_{\alpha_{2}}^{\beta_{2}} \int_{\alpha_{3}}^{\beta_{2}} \rho\left(\frac{1-\lambda_{1}-\lambda_{2}-\lambda_{3}}{\beta_{0}}\right) \frac{d \lambda_{1}}{\lambda_{1}} \frac{d \lambda_{2}}{\lambda_{2}} \frac{d \lambda_{3}}{\lambda_{3}} .
\end{aligned}
$$

Сделаем замену переменных $\mu_{1}=\lambda_{1}, \mu_{2}=\lambda_{1}+\lambda_{2}, \mu_{3}=\lambda_{1}+\lambda_{2}+\lambda_{3}$. Тогда

$$
G_{2}^{(1)}\left(\beta_{0}, \alpha_{1}, \beta_{1}, \alpha_{2}, \beta_{2}\right)=\int_{\alpha_{1}}^{\beta_{1}} \int_{\alpha_{2}+\mu_{1}}^{\beta_{2}+\mu_{1}} \rho\left(\frac{1-\mu_{2}}{\beta_{0}}\right) \frac{d \mu_{1}}{\mu_{1}} \frac{d \mu_{2}}{\mu_{2}-\mu_{1}}
$$

и

$$
\begin{aligned}
& G_{3}^{(1)}\left(\beta_{0}, \alpha_{1}, \beta_{1}, \alpha_{2}, \beta_{2}\right)= \\
& \quad=\frac{1}{2} \int_{\alpha_{1}}^{\beta_{1}} \int_{\alpha_{2}+\mu_{1}}^{\beta_{2}+\mu_{1}} \int_{\alpha_{2}+\mu_{2}}^{\beta_{2}+\mu_{2}} \rho\left(\frac{1-\mu_{3}}{\beta_{0}}\right) \frac{d \mu_{1}}{\mu_{1}} \frac{d \mu_{2}}{\mu_{2}-\mu_{1}} \frac{d \mu_{3}}{\mu_{3}-\mu_{2}} .
\end{aligned}
$$

Изменим порядок интегрирования по $\mu_{1}$ и $\mu_{2}$, а вслед за этим применим лемму 4. Тогда

$$
\begin{aligned}
& G_{2}^{(1)}\left(\beta_{0}, \alpha_{1}, \beta_{1}, \alpha_{2}, \beta_{2}\right)= \\
& \quad=\sum_{u, v} e(u, v) \int_{u+\alpha_{1}}^{v+\beta_{1}} d \mu_{2} \int_{l_{u}\left(\mu_{2}\right)}^{l_{v}\left(\mu_{2}\right)} \rho\left(\frac{1-\mu_{2}}{\beta_{0}}\right) \frac{1}{\mu_{1}\left(\mu_{1}-\mu_{2}\right)} d \mu_{1}
\end{aligned}
$$

и

$$
\begin{aligned}
& G_{3}^{(1)}\left(\beta_{0}, \alpha_{1}, \beta_{1}, \alpha_{2}, \beta_{2}\right)= \\
& =\frac{1}{2} \sum_{u, v} e(u, v) \int_{u+\alpha_{1}}^{v+\beta_{1}} d \mu_{2} \int_{l_{u}\left(\mu_{2}\right)}^{l_{v}\left(\mu_{2}\right)} \frac{d \mu_{1}}{\mu_{1}\left(\mu_{1}-\mu_{2}\right)} \int_{\alpha_{2}+\mu_{2}}^{\beta_{2}+\mu_{2}} \rho\left(\frac{1-\mu_{3}}{\beta_{0}}\right) \frac{d \mu_{3}}{\mu_{3}-\mu_{2}} .
\end{aligned}
$$


Здесь $p=q=1$, поэтому

$$
\begin{gathered}
e(u, v)=\left\{\begin{array}{lll}
-1 & \text { при } & (u, v)=\left(\alpha_{2}, \alpha_{2}\right), \\
+1 & \text { при } & (u, v)=\left(\beta_{2}, \alpha_{2}\right), \\
-1 & \text { при } & (u, v)=\left(\beta_{2}, \beta_{2}\right), \\
0 & \text { в остальных случаях, }
\end{array}\right. \\
l_{u}\left(\mu_{2}\right)=\left\{\begin{array}{lll}
\mu_{2}-\alpha_{2} & \text { при } & u=\alpha_{2}, \\
\beta_{1} & \text { при } & u=\beta_{2},
\end{array} \quad l_{v}\left(\mu_{2}\right)=\left\{\begin{array}{lll}
\alpha_{1} & \text { при } & v=\alpha_{2}, \\
\mu_{2}-\beta_{2} & \text { при } & v=\beta_{2} .
\end{array}\right.\right.
\end{gathered}
$$

Перепишем полученные равенства в следующем виде:

$$
G_{2}^{(1)}\left(\beta_{0}, \alpha_{1}, \beta_{1}, \alpha_{2}, \beta_{2}\right)=\sum_{u, v} e(u, v) \int_{u+\alpha_{1}}^{v+\beta_{1}} \rho\left(\frac{1-\mu_{2}}{\beta_{0}}\right) I\left(\mu_{2}\right) d \mu_{2}
$$

и

$$
\begin{aligned}
& G_{3}^{(1)}\left(\beta_{0}, \alpha_{1}, \beta_{1}, \alpha_{2}, \beta_{2}\right)= \\
& =\frac{1}{2} \sum_{u, v} e(u, v) \int_{u+\alpha_{1}}^{v+\beta_{1}} I\left(\mu_{2}\right) d \mu_{2} \int_{\alpha_{2}+\mu_{2}}^{\beta_{2}+\mu_{2}} \rho\left(\frac{1-\mu_{3}}{\beta_{0}}\right) \frac{d \mu_{3}}{\mu_{3}-\mu_{2}} .
\end{aligned}
$$

где

$$
I\left(\mu_{2}\right)=\int_{l_{u}\left(\mu_{2}\right)}^{l_{v}\left(\mu_{2}\right)} \frac{d \mu_{1}}{\mu_{1}\left(\mu_{1}-\mu_{2}\right)} .
$$

К выражению внутри последней суммы можно снова применить лемму 4. Тогда получим

$$
\begin{aligned}
& G_{3}^{(1)}\left(\beta_{0}, \alpha_{1}, \beta_{1}, \alpha_{2}, \beta_{2}\right)= \\
& =\frac{1}{2} \sum_{u, v} e(u, v) \sum_{u_{1}, v_{1}} e^{\prime}\left(u_{1}, v_{1}\right) \int_{u_{1}+u+\alpha_{1}}^{v_{1}+v+\beta_{1}} d \mu_{3} \int_{l_{u_{1}}^{\prime}\left(\mu_{3}\right)}^{l_{v_{1}}^{\prime}\left(\mu_{3}\right)} I\left(\mu_{2}\right) \frac{d \mu_{2}}{\mu_{3}-\mu_{2}} \rho\left(\frac{1-\mu_{3}}{\beta_{0}}\right),
\end{aligned}
$$

где штрихи во внутренней сумме всюду обозначают, что соответствующие величины надо рассматривать по отношению к параметрам $\alpha_{1}^{\prime}=u+\alpha_{1}$ и $\beta_{1}^{\prime}=v+\beta_{1}$, зависящим от индексов суммирования внешней суммы. 
Таким образом, $e^{\prime}(u, v)=e(u, v)$,

$l_{u_{1}}^{\prime}\left(\mu_{2}\right)=\left\{\begin{array}{lll}\mu_{2}-\alpha_{2} & \text { при } & u_{1}=\alpha_{2}, \\ v+\beta_{1} & \text { при } & u_{1}=\beta_{2},\end{array} \quad l_{v_{1}}^{\prime}\left(\mu_{2}\right)=\left\{\begin{array}{lll}u+\alpha_{1} & \text { при } & v_{1}=\alpha_{2}, \\ \mu_{2}-\beta_{2} & \text { при } & v_{1}=\beta_{2} .\end{array}\right.\right.$

Интеграл $I\left(\mu_{2}\right)$ можно заменить выражением, полученным в лемме 5. Учитывая, что $p=q=1$, приходим к формуле

$$
I\left(\mu_{2}\right)=\frac{1}{\mu_{2}}\left(\varepsilon_{1}(u, v) \ln \frac{\mu_{2}-\gamma_{1}(u, v)}{\gamma_{1}(u, v)}+\varepsilon_{2}(u, v) \ln \frac{\mu_{2}-\gamma_{2}(u, v)}{\gamma_{2}(u, v)}\right),
$$

где

$$
\begin{gathered}
\varepsilon_{0}(u, v)=\left\{\begin{array}{rrl}
-1 & \text { при } & u=\alpha_{2}, \\
+1 & \text { при } & u=\beta_{2}, \\
0 & \text { при } & u \notin\left\{\alpha_{2}, \beta_{2}\right\},
\end{array}\right. \\
\gamma_{0}(u, v)=\left\{\begin{array}{llll}
\alpha_{2} & \text { при } & u=\alpha_{2}, \\
\beta_{1} & \text { при } & u=\beta_{2},
\end{array} \quad \varepsilon_{1}(u, v)=\left\{\begin{array}{rrl}
-1 & \text { при } & v=\alpha_{2}, \\
+1 & \text { при } & v=\beta_{2}, \\
0 & \text { при } & v \notin\left\{\alpha_{2}, \beta_{2}\right\},
\end{array}\right.\right.
\end{gathered}
$$

Таким образом, доказана следующая теорема.

Теорема 2. В приведенных выше обозначениях имеют место формульь

$$
\begin{aligned}
& G_{2}^{(1)}\left(\beta_{0}, \alpha_{1}, \beta_{1}, \alpha_{2}, \beta_{2}\right)= \\
& =\sum_{u, v} e(u, v) \int_{u+\alpha_{1}}^{v+\beta_{1}} \rho\left(\frac{1-\mu_{2}}{\beta_{0}}\right)\left(\varepsilon_{1}(u, v) \ln \frac{\mu_{2}-\gamma_{1}(u, v)}{\gamma_{1}(u, v)}+\right. \\
& \left.+\varepsilon_{2}(u, v) \ln \frac{\mu_{2}-\gamma_{2}(u, v)}{\gamma_{2}(u, v)}\right) \frac{d \mu_{2}}{\mu_{2}}
\end{aligned}
$$

$u$

$$
\begin{aligned}
& G_{3}^{(1)}\left(\beta_{0}, \alpha_{1}, \beta_{1}, \alpha_{2}, \beta_{2}\right)= \\
& =\frac{1}{2} \sum_{u, v} e(u, v) \sum_{u_{1}, v_{1}} e^{\prime}\left(u_{1}, v_{1}\right) \times \\
& \quad \times \int_{u_{1}+u+\alpha_{1}}^{v_{1}+v+\beta_{1}} \rho\left(\frac{1-\mu_{3}}{\beta_{0}}\right) d \mu_{3} \int_{l_{u_{1}}^{\prime}\left(\mu_{3}\right)}^{l_{v_{1}}^{\prime}\left(\mu_{3}\right)}\left(\varepsilon_{1}(u, v) \ln \frac{\mu_{2}-\gamma_{1}(u, v)}{\gamma_{1}(u, v)}+\right. \\
& \left.\quad+\varepsilon_{2}(u, v) \ln \frac{\mu_{2}-\gamma_{2}(u, v)}{\gamma_{2}(u, v)}\right) \frac{d \mu_{2}}{\mu_{2}\left(\mu_{3}-\mu_{2}\right)} .
\end{aligned}
$$


Теперь рассмотрим интегралы

$$
G_{2}^{(2)}\left(\beta_{0}, \alpha_{1}, \beta_{1}, \beta_{2}\right)=\int_{\alpha_{1}}^{\beta_{1}} \int_{\lambda_{1}}^{\beta_{2}} \rho\left(\frac{1-\lambda_{1}-\lambda_{2}}{\beta_{0}}\right) \frac{d \lambda_{1}}{\lambda_{1}} \frac{d \lambda_{2}}{\lambda_{2}}
$$

и

$$
\begin{aligned}
& G_{3}^{(2)}\left(\beta_{0}, \alpha_{1}, \beta_{1}, \beta_{2}\right)= \\
& \quad=\frac{1}{2} \int_{\alpha_{1}}^{\beta_{1}} \int_{\lambda_{1}}^{\beta_{2}} \int_{\lambda_{1}}^{\beta_{2}} \rho\left(\frac{1-\lambda_{1}-\lambda_{2}-\lambda_{3}}{\beta_{0}}\right) \frac{d \lambda_{1}}{\lambda_{1}} \frac{d \lambda_{2}}{\lambda_{2}} \frac{d \lambda_{3}}{\lambda_{3}} .
\end{aligned}
$$

Сделаем замену переменных $\mu_{1}=\lambda_{1}, \mu_{2}=\lambda_{1}+\lambda_{2}, \mu_{3}=\lambda_{1}+\lambda_{2}+\lambda_{3}$. Тогда

$$
G_{2}^{(2)}\left(\beta_{0}, \alpha_{1}, \beta_{1}, \beta_{2}\right)=\int_{\alpha_{1}}^{\beta_{1}} \int_{2 \mu_{1}}^{\beta_{2}+\mu_{1}} \rho\left(\frac{1-\mu_{2}}{\beta_{0}}\right) \frac{d \mu_{1}}{\mu_{1}} \frac{d \mu_{2}}{\mu_{2}-\mu_{1}}
$$

и

$$
\begin{aligned}
& G_{3}^{(2)}\left(\beta_{0}, \alpha_{1}, \beta_{1}, \beta_{2}\right)= \\
& \quad=\frac{1}{2} \int_{\alpha_{1}}^{\beta_{1}} \int_{2 \mu_{1}}^{\beta_{2}+\mu_{1}} \int_{\mu_{1}+\mu_{2}}^{\beta_{2}+\mu_{2}} \rho\left(\frac{1-\mu_{3}}{\beta_{0}}\right) \frac{d \mu_{1}}{\mu_{1}} \frac{d \mu_{2}}{\mu_{2}-\mu_{1}} \frac{d \mu_{3}}{\mu_{3}-\mu_{2}} .
\end{aligned}
$$

Применим лемму 4. Тогда

$$
\begin{aligned}
& G_{2}^{(2)}\left(\beta_{0}, \alpha_{1}, \beta_{1}, \beta_{2}\right)= \\
& =\sum_{u, v} e(u, v) \int_{u+\alpha_{1}}^{v+\beta_{1}} \int_{l_{u}\left(\mu_{2}\right)}^{l_{v}\left(\mu_{2}\right)} \rho\left(\frac{1-\mu_{2}}{\beta_{0}}\right) \frac{d \mu_{1} d \mu_{2}}{\mu_{1}\left(\mu_{1}-\mu_{2}\right)}
\end{aligned}
$$

и

$$
\begin{aligned}
& G_{3}^{(2)}\left(\beta_{0}, \alpha_{1}, \beta_{1}, \beta_{2}\right)= \\
& =\frac{1}{2} \sum_{u, v} e(u, v) \int_{u+\alpha_{1}}^{v+\beta_{1}} d \mu_{2} \int_{l_{u}\left(\mu_{2}\right)}^{l_{v}\left(\mu_{2}\right)} d \mu_{1} \int_{\mu_{1}+\mu_{2}}^{\beta_{2}+\mu_{2}} \rho\left(\frac{1-\mu_{3}}{\beta_{0}}\right) \frac{d \mu_{3}}{\mu_{1}\left(\mu_{2}-\mu_{1}\right)\left(\mu_{2}-\mu_{1}\right)} .
\end{aligned}
$$

Здесь $\alpha_{2}=0, p=2$ и $q=1$, поэтому

$$
e(u, v)=\left\{\begin{array}{rll}
-1 & \text { при } & (u, v)=\left(\alpha_{1}, \beta_{1}\right) \\
+1 & \text { при } & (u, v)=\left(\beta_{2}, \beta_{1}\right) \\
-1 & \text { при } & (u, v)=\left(\beta_{2}, \beta_{2}\right) \\
0 & \text { в остальных случаях }
\end{array}\right.
$$




$$
l_{u}\left(\mu_{2}\right)=\left\{\begin{array}{lll}
\frac{\mu_{2}}{2} & \text { при } & u=\alpha_{1}, \\
\beta_{1} & \text { при } & u=\beta_{2},
\end{array} \quad l_{v}\left(\mu_{2}\right)=\left\{\begin{array}{lll}
\alpha_{1} & \text { при } & v=\beta_{2}, \\
\mu_{2}-\beta_{2} & \text { при } & v=\beta_{2} .
\end{array}\right.\right.
$$

В полученном выражении для $G_{2}^{(2)}$ внутренний интеграл можно подсчитать при помощи леммы 5. С учетом того, что $\alpha_{2}=0, p=2$ и $q=1$, приведенная в формулировке леммы формула упрощается следующим образом:

$$
\begin{aligned}
& \int_{l_{u}\left(\mu_{2}\right)}^{l_{v}\left(\mu_{2}\right)} \frac{d \mu_{1}}{\mu_{1}\left(\mu_{2}-\mu_{1}\right)}= \\
& \quad=\frac{1}{\mu_{2}}\left(\varepsilon_{1}(u, v) \ln \frac{\mu_{2}-\gamma_{1}(u, v)}{\gamma_{1}(u, v)}+\varepsilon_{2}(u, v) \ln \frac{\mu_{2}-\gamma_{2}(u, v)}{\gamma_{2}(u, v)}\right),
\end{aligned}
$$

где

$$
\begin{gathered}
\varepsilon_{0}(u, v)=\left\{\begin{array}{rll}
+1 & \text { при } & u=\beta_{2}, \\
0 & \text { при } & u \neq \beta_{2},
\end{array} \quad \varepsilon_{1}(u, v)=\left\{\begin{array}{rll}
-1 & \text { при } & v=\beta_{1}, \\
+1 & \text { при } & v=\beta_{2}, \\
0 & \text { при } & v \notin\left\{\beta_{1}, \beta_{2}\right\},
\end{array}\right.\right. \\
\gamma_{0}(u, v)=\beta_{1}, \quad \gamma_{1}(u, v)=\left\{\begin{array}{lll}
\alpha_{1} & \text { при } & v=\beta_{1}, \\
\beta_{2} & \text { при } & v=\beta_{2} .
\end{array}\right.
\end{gathered}
$$

Действительно, согласно доказательству леммы 5 в случае $(u, v)=\left(\alpha_{1}, \beta_{1}\right)$ рассматриваемый интеграл равен

$$
\int_{l_{u}\left(\mu_{2}\right)}^{l_{v}\left(\mu_{2}\right)} \frac{d \mu_{1}}{\mu_{1}\left(\mu_{2}-\mu_{1}\right)}=\frac{1}{\mu_{2}} \ln \frac{\alpha_{1}\left(\mu_{2}-\mu_{2} / 2\right)}{\left.\left(\mu_{2}-\alpha_{1}\right) \mu_{2} / 2\right)}=\frac{1}{\mu_{2}} \ln \frac{\alpha_{1}}{\mu_{2}-\alpha_{1}}
$$

в случае $(u, v)=\left(\beta_{2}, \beta_{1}\right)$ он равен

$$
\frac{1}{\mu_{2}} \ln \frac{\alpha_{1}\left(\mu_{2}-\beta_{1}\right)}{\left(\mu_{2}-\alpha_{1}\right) \beta_{1}}
$$

а в случае $(u, v)=\left(\beta_{2}, \beta_{1}\right)$ он равен

$$
\frac{1}{\mu_{2}} \ln \frac{\left(\mu_{2}-\beta_{2}\right)\left(\mu_{2}-\beta_{1}\right)}{\mu_{2}-\left(\mu_{2}-\beta_{2}\right) \beta_{1}}=\frac{1}{\mu_{2}} \ln \frac{\left(\mu_{2}-\beta_{2}\right)\left(\mu_{2}-\beta_{1}\right)}{\beta_{2} \beta_{1}} .
$$


Очевидно, что во всех трех случаях полученное выражение совпадает с правой частью равенства (6).

Применяя равенство (6) к интегралу $G_{2}^{(2)}$, получаем

$$
\begin{array}{r}
G_{2}^{(2)}\left(\beta_{0}, \alpha_{1}, \beta_{1}, \beta_{2}\right)=\sum_{u, v} e(u, v) \int_{u+\alpha_{1}}^{v+\beta_{1}} \rho\left(\frac{1-\mu_{2}}{\beta_{0}}\right)\left(\varepsilon_{1}(u, v) \ln \frac{\mu_{2}-\gamma_{1}(u, v)}{\gamma_{1}(u, v)}+\right. \\
\left.+\varepsilon_{2}(u, v) \ln \frac{\mu_{2}-\gamma_{2}(u, v)}{\gamma_{2}(u, v)}\right) \frac{d \mu_{2}}{\mu_{2}} .
\end{array}
$$

В случае $G_{3}^{(2)}$ возникают некоторые трудности по сравнению с $G_{3}^{(1)}$ и $G_{2}^{(2)}$, поскольку здесь имеется неприятная зависимость от $\mu_{1}$ в нижнем пределе интегрирования последнего из повторных интегралов.

Применим формулу интегрирования по частям $\int F^{\prime} \Phi d \mu_{1}=F \varphi-\int F \Phi^{\prime} d \mu_{1}$, полагая

$$
\begin{aligned}
& F\left(\mu_{1}\right)=\int \frac{d \mu_{1}}{\mu_{1}\left(\mu_{2}-\mu_{1}\right)}=\frac{1}{\mu_{2}} \ln \frac{\mu_{1}}{\mu_{2}-\mu_{1}}, \\
& \Phi\left(\mu_{1}\right)=\int_{\mu_{1}+\mu_{2}}^{\beta_{2}+\mu_{2}} \rho\left(\frac{1-\mu_{3}}{\beta_{0}}\right) \frac{d \mu_{3}}{\mu_{3}-\mu_{2}} .
\end{aligned}
$$

Тогда получим

$$
\begin{aligned}
& \int_{l_{u}\left(\mu_{2}\right)}^{l_{v}\left(\mu_{2}\right)} \frac{d \mu_{1}}{\mu_{1}\left(\mu_{2}-\mu_{1}\right)} \int_{\mu_{1}+\mu_{2}}^{\beta_{2}+\mu_{2}} \rho\left(\frac{1-\mu_{3}}{\beta_{0}}\right) \frac{d \mu_{3}}{\mu_{3}-\mu_{2}}= \\
& =\left.\left(\frac{1}{\mu_{2}} \ln \frac{\mu_{1}}{\mu_{2}-\mu_{1}} \int_{\mu_{1}+\mu_{2}}^{\beta_{2}+\mu_{2}} \rho\left(\frac{1-\mu_{3}}{\beta_{0}}\right) \frac{d \mu_{3}}{\mu_{3}-\mu_{2}}\right)\right|_{\mu_{1}=l_{u}\left(\mu_{2}\right)} ^{\mu_{1}=l_{v}\left(\mu_{2}\right)}+ \\
& \quad+\int_{l_{u}\left(\mu_{2}\right)}^{l_{v}\left(\mu_{2}\right)} \rho\left(\frac{1-\mu_{1}-\mu_{2}}{\beta_{0}}\right) \frac{1}{\mu_{2}} \ln \frac{\mu_{1}}{\mu_{2}-\mu_{1}} \frac{d \mu_{1}}{\mu_{1}} .
\end{aligned}
$$

В последнем интеграле сделаем замену переменной $\mu_{3}=\mu_{1}+\mu_{2}$. Тогда

$$
\begin{aligned}
& \int_{l_{u}\left(\mu_{2}\right)}^{l_{v}\left(\mu_{2}\right)} \frac{d \mu_{1}}{\mu_{1}\left(\mu_{2}-\mu_{1}\right)} \int_{\mu_{1}+\mu_{2}}^{\beta_{2}+\mu_{2}} \rho\left(\frac{1-\mu_{3}}{\beta_{0}}\right) \frac{d \mu_{3}}{\mu_{3}-\mu_{2}}= \\
& =\left.\left(\frac{1}{\mu_{2}} \ln \frac{\mu_{1}}{\mu_{2}-\mu_{1}} \int_{\mu_{1}+\mu_{2}}^{\beta_{2}+\mu_{2}} \rho\left(\frac{1-\mu_{3}}{\beta_{0}}\right) \frac{d \mu_{3}}{\mu_{3}-\mu_{2}}\right)\right|_{\mu_{1}=l_{u}\left(\mu_{2}\right)} ^{\mu_{1}=l_{v}\left(\mu_{2}\right)}+ \\
& \quad+\int_{l_{u}\left(\mu_{2}\right)+\mu_{2}}^{l_{v}\left(\mu_{2}\right)+\mu_{2}} \rho\left(\frac{1-\mu_{3}}{\beta_{0}}\right) \frac{1}{\mu_{2}} \ln \frac{\mu_{2}-\mu_{1}}{2 \mu_{2}-\mu_{3}} \frac{d \mu_{3}}{\mu_{3}-\mu_{2}} .
\end{aligned}
$$


Напомним, что функции $l_{u}\left(\mu_{2}\right)$ и $l_{v}\left(\mu_{2}\right)$ в данном случае (при $\alpha_{2}=0, p=2$ и $q=1)$ имеют вид

$$
l_{u}\left(\mu_{2}\right)=\left\{\begin{array}{lll}
\frac{\mu_{2}}{2} & \text { при } & u=\alpha_{1}, \\
\beta_{1} & \text { при } & u=\beta_{2},
\end{array} \quad l_{v}\left(\mu_{2}\right)=\left\{\begin{array}{lll}
\alpha_{1} & \text { при } & v=\beta_{2}, \\
\mu_{2}-\beta_{2} & \text { при } & v=\beta_{2} .
\end{array}\right.\right.
$$

Это показывает, что равенство (8) можно переписать в виде

$$
\begin{gathered}
\int_{l_{u}\left(\mu_{2}\right)}^{l_{v}\left(\mu_{2}\right)} \frac{d \mu_{1}}{\mu_{1}\left(\mu_{2}-\mu_{1}\right)} \int_{\mu_{1}+\mu_{2}}^{\beta_{2}+\mu_{2}} \rho\left(\frac{1-\mu_{3}}{\beta_{0}}\right) \frac{d \mu_{3}}{\mu_{3}-\mu_{2}}= \\
=\sum_{i=0}^{2} \int_{J_{i}} \rho\left(\frac{1-\mu_{3}}{\beta_{0}}\right) \frac{d \mu_{3}}{\mu_{3}-\mu_{2}} \frac{1}{\mu_{2}} \ln \frac{L_{1}^{(i)}\left(\mu_{2}, \mu_{3}\right)}{L_{2}^{(i)}\left(\mu_{2}, \mu_{3}\right)}
\end{gathered}
$$

где $J_{i}=\left[a_{u, v}^{(i)}+p_{u, v}^{(i)} \mu_{2}, a_{u, v}^{(i+1 \bmod 3)}+p_{u, v}^{(i+1 \bmod 3)} \mu_{2}\right], i=0,1,2, a_{u, v}^{(0)}=\beta_{2}, p_{u, v}^{(0)}=1$,

$$
\begin{aligned}
& a_{u, v}^{(1)}=\left\{\begin{array}{lll}
\alpha_{1} & \text { при } & u=\alpha_{1}, \\
\beta_{1} & \text { при } & u=\beta_{2},
\end{array} \quad a_{u, v}^{(2)}=\left\{\begin{array}{lll}
\alpha_{1} & \text { при } & v=\beta_{1}, \\
-\beta_{2} & \text { при } & v=\beta_{2} .
\end{array}\right.\right. \\
& p_{u, v}^{(1)}=\left\{\begin{array}{rrr}
\frac{3}{2} & \text { при } & u=\alpha_{1}, \\
1 & \text { при } & u=\beta_{2},
\end{array} \quad p_{u, v}^{(2)}=\left\{\begin{array}{ccc}
1 & \text { при } & v=\beta_{1}, \\
1 & \text { при } & v=\beta_{2},
\end{array}\right.\right. \\
& L_{1}^{(0)}=l_{u}\left(\mu_{2}\right), \quad L_{2}^{(0)}=\mu_{2}-l_{u}\left(\mu_{2}\right), \\
& L_{1}^{(2)}=l_{v}\left(\mu_{2}\right), \quad L_{2}^{(2)}=\mu_{2}-l_{v}\left(\mu_{2}\right) \text {, } \\
& L_{1}^{(1)}=\mu_{3}-\mu_{2}, \quad L_{2}^{(1)}=2 \mu_{2}-\mu_{3} .
\end{aligned}
$$

Таким образом, выражение для $G_{3}^{(2)}$ принимает вид

$$
\begin{aligned}
& G_{3}^{(2)}\left(\beta_{0}, \alpha_{1}, \beta_{1}, \beta_{2}\right)= \\
& =\frac{1}{2} \sum_{i=0}^{2} \sum_{u, v} e(u, v) \int_{u+\alpha_{1}}^{v+\beta_{1}} \frac{d \mu_{2}}{\mu_{2}} \int_{J_{i}} \rho\left(\frac{1-\mu_{3}}{\beta_{0}}\right) \frac{d \mu_{3}}{\mu_{3}-\mu_{2}} \frac{1}{\mu_{2}} \ln \frac{L_{1}^{(i)}\left(\mu_{2}, \mu_{3}\right)}{L_{2}^{(i)}\left(\mu_{2}, \mu_{3}\right)} .
\end{aligned}
$$

К выражению внутри последней суммы можно снова применить лемму 4. Тогда получим формулу (9) ниже.

Таким образом, доказана следующая теорема. 
Теорема 3. Для интегралов $G_{2}^{(2)}\left(\beta_{0}, \alpha_{1}, \beta_{1}, \beta_{2}\right)$ и $G_{3}^{(2)}\left(\beta_{0}, \alpha_{1}, \beta_{1}, \beta_{2}\right)$ в приведенных выше обозначениях имеют место выражения

$$
\begin{aligned}
& \sum_{u, v} e(u, v) \int_{u+\alpha_{1}}^{v+\beta_{1}} \rho\left(\frac{1-\mu_{2}}{\beta_{0}}\right)\left(\varepsilon_{1}(u, v) \ln \frac{\mu_{2}-\gamma_{1}(u, v)}{\gamma_{1}(u, v)}+\right. \\
& \left.\quad+\varepsilon_{2}(u, v) \ln \frac{\mu_{2}-\gamma_{2}(u, v)}{\gamma_{2}(u, v)}\right) \frac{d \mu_{2}}{\mu_{2}} \\
& \begin{aligned}
\frac{1}{2} \sum_{i=0}^{2} \sum_{u, v} e(u, v) \sum_{u_{1}, v_{1}} e^{\prime}\left(u_{1}, v_{1}\right) \times \\
\quad \times \int_{u_{1}+u+\alpha_{1}}^{v_{1}+v+\beta_{1}} d \mu_{3} \int_{l_{u_{1}}^{\prime}\left(\mu_{3}\right)}^{l_{v_{1}}^{\prime}\left(\mu_{3}\right)} \rho\left(\frac{1-\mu_{3}}{\beta_{0}}\right) \frac{d \mu_{2}}{\mu_{2}\left(\mu_{3}-\mu_{2}\right)} \ln \frac{L_{1}^{(i)}\left(\mu_{2}, \mu_{3}\right)}{L_{2}^{(i)}\left(\mu_{2}, \mu_{3}\right)},
\end{aligned}
\end{aligned}
$$

где штрихи во внутренней сумме всюду обозначают, что соответствующие величины надо рассматривать по отнотению к параметрам $\alpha_{1}^{\prime}=u+\alpha_{1}$, $\beta_{1}^{\prime}=v+\beta_{1}, \alpha_{2}^{\prime}=a_{u, v}^{(i)}, \beta_{2}^{\prime}=a_{u, v}^{(i+1 \bmod 3)}, p^{\prime}=p_{u, v}^{(i)}, q^{\prime}=p_{u, v}^{(i+1 \bmod 3)}, i=0,1,2$, зависящим от индексов суммирования внешних сумм.

\section{Список литературы}

[1] Рыбаков А. С., “Модификация алгоритма оценки количества целых чисел, имеющих не более трех больших простых делителей”, Матем. вопросы криптографии, 4:3 (2013), $131-158$.

[2] Aoki K., Bos J.W., Franke J., Gaudry P., Kleinjung T., Kruppa A., Lenstra A. K., Montgomery P. L., Osvik D. A., te Riele H., Thome E., Timofeev A., Zimmermann P., "Factorization of a 768-bit RSA modulus" (2010), http://www.loria.fr/ zimmerma/records/rsa768.

[3] Bach E., Peralta R., “Asymptotic semismoothness probabilities”, Math. Comput., 65 :216 (1996), $1701-1715$.

[4] Ekkelkamp W.H., On the Amount of Sieving in Factorization Methods, Ph. D. thesis, Leiden: Univ. Leiden/CWI/T. Stieltjes Inst. Math., 2010, iv+111 pp.

[5] Knuth D. E., Pardo L. T., "Analysis of a simple factorization algorithm", Theor. Comput. Sci., 3:3 (1976), 321-348.

[6] Lambert R. J., Computational Aspects of Discrete Logarithms, Ph. D. thesis, Ontario: Univ. Waterloo, Electrical Engineering, 1996, http://www.cacr.math.uwaterloo.ca/techreports/2000/ lambert-thesis.ps.

[7] Pollard J. M., "The lattice sieve". In: "The Development of the Number Field Sieve”, Lect. Notes Math, 1554 / под общ. ред. Lenstra A. K., Lenstra H. W., Jr., 1993, 43-49.

\section{МАТЕМАТИЧЕСКИЕ ВОПРОСЫ КРИПТОГРАФИИ}

